\title{
Oscillatory and Asymptotic Properties of the Solutions of a Class of Operator-Differential Equations
}

\author{
D.D. BAINOV ${ }^{1}$, M.B. DIMITROVA ${ }^{2}$ and A.D. MYSHKIS
}

ABSTRACT. In the present paper the oscillatory and asymtotic properties of the solutions of the operator-differential equation

$$
\left[\tau_{n-1}(t)\left[\tau_{n-2}(t)\left[\ldots\left[\tau_{1}(t)\left[\tau_{0}(t) \cdot x(t)\right]^{\prime}\right]^{\prime} \ldots\right]^{\prime}\right]^{\prime}\right]^{\prime}+\delta \cdot(A x)(t)=0
$$

are investigated, where $A$ is a monotonic operator with certain properties.

Particular realizations of the operator $A$ are given, for which the results obtained can be applied.

\footnotetext{
1,2 Supported by the Bulgarian Ministry of Science and Higher Education under
} Grant MM-7

1991 Mathematics Subject Classification: 34C10, 34D05

Editorial Complutense. Madrid, 1994. 


\section{INTRODUCTION}

In 1987 the book of Ladde, Lakshmikantham, Zhang [2] was published. In it for the first time in sufficient details problems related to the oscillation and asymptotic theory of functional differential equations are considered. Parallel to the development of the oscillation theory of functional differential equations the development of the oscillation and asymptotic theory of various classes of ordinary differential equations began, such as differential equations with "maxima", impulsive differential equations, integro-differential equations, etc. We shall note that the results obtained for these equations are of isolated character and the traditional problems set in the oscillation theory are almost untouched for them.

In the present paper the oscillatory and asymptotic properties of the solutions of a class of homogeneous operator-differential equations are investigated and thus by means of a single approach the properties of the solutions of numerous little investigated classes of differential equations are studied. We shall note that an analogous approach was used in Mishev, Bainov [3].

\section{PRELIMINARY NOTES}

Consider the operator-differential equation

$$
\left[\tau_{n-1}(t)\left[\tau_{n-2}(t)\left[\ldots\left[\tau_{1}(t)\left[\tau_{0}(t), x(t)\right]^{\prime}\right]^{\prime} \ldots\right]^{\prime}\right]^{\prime}\right]^{\prime}+\delta \cdot(A x)(t)=0
$$

where $\delta= \pm 1, n \geq 1$; the number $t_{0} \in R$ is fixed. $A$ is an operator with certain properties: $\tau_{i} \in C\left(\left[t_{0}, \infty\right),(0, \infty)\right), i=0, \ldots, n-1$. Here $C(M, N)$ is the set of all continuous functions $f: M \rightarrow N$.

Introduce the following notation:

$$
\begin{aligned}
\left(L_{0} x\right)(t) & =\tau_{0}(t) \cdot x(t) \\
\left(L_{i} x\right)(t) & =\tau_{i}(t)\left[\left(L_{i-1} x\right)(t)\right]^{\prime}, i=1, \ldots, n, \tau_{n}: \equiv 1 \\
R_{0}(t) & =1 \\
R_{1}(t) & =\int_{t_{0}}^{t} \frac{d s_{1}}{\tau_{1}\left(s_{1}\right)}
\end{aligned}
$$




$$
\begin{gathered}
\left.R_{i}(t)=\int_{t_{0}}^{t} \frac{d s_{1}}{\tau_{1}\left(s_{1}\right)}\left(\int_{t_{0}}^{s_{1}} \frac{d s_{2}}{\tau_{2}\left(s_{2}\right)}\left(\cdots\left(\int_{t_{0}}^{s_{i-1}} \frac{d s_{i}}{\tau_{i}\left(s_{i}\right)}\right) \cdots\right)\right)\right) \\
i=2, \ldots, n-1 \text { for } n>2
\end{gathered}
$$

Denote by $D_{n}$ the set of all functions $x \in C\left(\left[T_{x}, \infty\right) ; R\right)\left(T_{x} \geq t_{0}\right)$ such that the functions $L_{i} x(i=0,1, \ldots, n)$ exist and are continuous for $\left[T_{x}, \infty\right)$.

Definition 1. The function $x:\left[T_{x}, \infty\right) \rightarrow R$ is said to be a solution of equation (1) if $x \in D_{n}$ and $x$ satisfies equation (1) for $t \geq$ $\max \left\{T_{x}, T_{A_{x}}\right\}\left(T_{A_{x}} \geq t_{0}\right)$.

Definition 2. A given function $u:\left[t_{0}, \infty\right) \rightarrow R$ is said to eventually enjoy the property $P$ if there exists a point $t_{p, u} \geq t_{0}$ such that for $t \geq t_{p, u}$ the property $P$ is valid.

Definition 3. The function $x \in C\left(\left[T_{x}, \infty\right) ; R\right)$ is said to be eventually zero if $x(t)=0$ eventually, and eventually nonzero otherwise.

Definition 4. The function $x \in C\left(\left[T_{x}, \infty\right) ; R\right)$ is said to oscillate if there exists a sequence of numbers $t_{1}<t_{2}<\cdots<t_{n}<\cdots, \lim _{n \rightarrow \infty} t_{n}=\infty$ such that $x\left(t_{i}\right) x\left(t_{i+1}\right)<0$. Otherwise the function is said to be nonoscillating. The function $x$ is said to weakly oscillate if $\sup \{t: x(t)=0\}=$ $\infty$.

For any nonoscillating function $y \in C\left(\left[T_{y}, \infty\right) ; R\right)\left(T_{y} \geq t_{0}\right)$ we define the function

$$
\begin{aligned}
\left(\psi_{n-1} y\right)(t) & =\int_{t}^{\infty} y(s) d s \\
\left(\psi_{i} y\right)(t) & =\int_{t}^{\infty} \frac{\left(\psi_{i+1} y\right)(s)}{\tau_{i+1}(s)} d s, i=n-2, \ldots, 0 ; n>1
\end{aligned}
$$

We shall say that conditions $(H)$ ane satisfied if the following conditions hold: 
$H 1 . \tau_{i} \in C\left(\left[t_{0}, \infty\right),(0, \infty)\right), i=0, \ldots, n-1$.

H2. $\int_{t_{0}}^{\infty} \frac{d t}{\tau_{i}(t)}=\infty, i=1, \ldots, n-1$.

$H 3 . A: D_{n} \rightarrow C\left(\left[T_{A x}, \infty\right) ; R\right)$.

H4. If the functions $x_{1}, x_{2} \in D_{n}$ and $x_{1}(t) \leq x_{2}(t)$ eventually, then $\left(A x_{1}\right)(t) \leq\left(A x_{2}\right)(t)$ eventually.

H5. If the function $x \in D_{n}$ and $x(t)=0$ eventually, then $\left(A_{x}\right)(t)=$ 0 eventually.

H6. If the function $x \in D_{n}$ and $x$ is eventually nonzero and nonoscillating, then the function $A x$ is eventually nonzero.

Lemma 1. Let the following conditions hold:

1. Conditions $\mathrm{H} 1$ and $\mathrm{H} 2$ hold.

2. The function $x \in D_{n}$.

3. The function $L_{n} x$ is eventually nonzero and nonoscillating.

Then:

1. Each function $L_{i} x(i=0, \ldots, n-1)$ is eventually monotonic and nonzero.

2. If $n>1$ and $\lim _{t \rightarrow \infty}\left(L_{i} x\right)(t) \neq 0$ for some $i=1, \ldots, n-1$, then $\lim _{t \rightarrow \infty}\left(L_{j} x\right)(t)=\operatorname{sgn}\left(\lim _{t \rightarrow \infty}\left(L_{i} x\right)(t)\right), \infty$ for any $j=0, \ldots, i-1$.

3. If $\lim _{t \rightarrow \infty}\left(L_{i} x\right)(t)=0$ for some $i=0, \ldots, n-1$, then $\left(L_{j} x\right) \cdot(t)$. $\left(L_{j+1} x\right)(t) \leq 0$ eventually for $j=1, \ldots, n-1$.

4. If $\left(L_{i} x\right)(t) \cdot\left(L_{i+1} x\right)(t) \leq 0$ eventually for some $i=0, \ldots, n-1$, then $\lim _{t \rightarrow \infty}\left(L_{i} x\right)(t)=C \in \mathbf{R}$.

5. If $\lim _{t \rightarrow \infty}\left(L_{i} x\right)(t) \in \mathbf{R}$ for some $i=0, \ldots, n-1$, then

$$
\begin{gathered}
\int_{t}^{\infty} \frac{\left|\left(L_{i+1} x\right)(s)\right|}{\tau_{i+1}(s)} d s<\infty \quad \text { and } \\
\left(L_{i} x\right)(t)=\lim _{t \rightarrow \infty}\left(L_{i} x\right)(t)+(-1)^{n-i}\left(\psi_{i} L_{n} x\right)(t) .
\end{gathered}
$$


6. For $n>1$ the following equality is valid

$$
\lim _{i \rightarrow \infty} \frac{\left(L_{0} x\right)(t)}{R_{i}(t)}=\lim _{t \rightarrow \infty}\left(L_{i} x\right)(t), i=1, \ldots, n-1 .
$$

Remark 1. Lemma 1 is a corollary of the respective theorems proved in [1], [5], [6], [7].

\section{MAIN RESULTS}

Theorem 1. Let the following conditions hold:

1. Conditions (H) are met.

2. For any constant $c \in \mathbf{R} \backslash\{0\}$ there exists an integer $i \in[0, n-1]$ such that eventually the following relation is valid

$$
\left|\left(\psi_{i} A \frac{c}{\tau_{0}}\right)(t)\right|=\infty
$$

Then for the existence of an eventually nonzero nonoscillating solution $x$ of equation (I) for which $L_{0} x$ is a bounded function it is necessary for $\delta=1(\delta=-1)$ that $n$ be an odd (even) number. For these solutions the following relations are valid

$$
\lim _{t \rightarrow \infty}\left(L_{i} x\right)(t)=0 \text { and } \operatorname{sgn}\left(L_{i} x\right)(t)=(-1)^{i} \operatorname{sgn} x(t)(i=0, \ldots, n-1)
$$

Proof. Let $x \in D_{n}$ be an eventually nonzero, nonoscillating solution of equation (1). Without loss of generality we can assume that $x(t) \geq 0$ for $t \in\left[t_{1}, \infty\right)$, where $t_{1} \geq t_{0}$. From condition $\mathrm{H} 4$ it follows that there exists a point $t_{2} \geq t_{1}$ such that $(A x)(t) \geq 0$ for $t \geq t_{0}$.

Then from equation (1) it follows that

$$
\delta\left(L_{n} x\right)(t)=-(A x)(t) \leq 0 \quad \text { for } t \geq t_{2}
$$


i.e. we can apply Lemma 1 .

We shall prove that $\lim _{t \rightarrow \infty}\left(L_{0} x\right)(t)=0$. If we suppose that this is not true, then from assertion 1 of Lemma 1 it follows that $\tau_{0}(t) \cdot x(t) \geq c>0$ eventually for some constant $c$.

From condition $\mathrm{H} 4$ and (1) it follows that eventually the following inequality holds

$$
\left|\left(L_{n} x\right)(t)\right|=(A x)(t) \geq\left(A \frac{c}{\tau_{0}}\right)(t)
$$

Choose $i(i=0, \ldots, n-1)$ which corresponds to the constant $c$ (see condition 2 of Theorem 1). From assertion 2 of Lemma 1 it follows that $\lim _{t \rightarrow \infty}\left(L_{i} x\right)(t)$ is a finite number. Then from equality (2) it follows that

$$
\left|\left(\psi_{i} L_{n} x\right)(t)\right|<\infty
$$

From inequalities (6) and (7) we obtain that

$$
\left|\left(\psi_{i} A \frac{c}{\tau_{0}}\right)(t)\right|<\infty
$$

which contradicts condition (4). Hence

$$
\lim _{t \rightarrow \infty}\left(L_{0} x\right)(t)=0
$$

From (8) and assertions 2 and 3 of Lemma 1 it follows that

$$
\begin{gathered}
\left(L_{j} x\right)(t) \cdot\left(L_{j+1} x\right)(t) \leq 0, j=0, \ldots, n-1 \\
\lim _{t \rightarrow \infty}\left(L_{j} x\right)(t)=0
\end{gathered}
$$

From inequalities (9) and (5) it follows that for $\delta=1(\delta=-1)$ the number $n$ is odd (even).

Theorem 2. Let the following conditions hold: 
1. Conditions (H) are met.

2. Condition 2 of Theorem 1 holds.

3. $\delta=1$.

4. For $n>1$ for any integer $i \in[0, n-2]$ which is odd or even just as $n$ is, and for any constant $c \in \mathbf{R} \backslash\{0\}$ the following relation is eventually valid

$$
\left|\left(\psi_{i+1} A \frac{c R_{i}}{\tau_{0}}\right)(t)\right|=\infty
$$

Then the assertion of Theorem 1 is valid without the requirement for boundedness of the function $L_{0} x$.

Proof. Let $x$ be an eventually nonzero nonoscillating solution of equation (1). Without loss of generality assume that $x(t) \geq 0$ eventually. Then from conditions $\mathrm{H} 4, \mathrm{H} 5$, condition 3 of Theorem 1 and equation (1) it follows that $\left(L_{n} x\right)(t) \leq 0$ eventually and we can apply Lemma 1 . Let $i$ be the greatest integer for which eventually the inequalities $\left(L_{i} x\right)(t)>0$ and $\left(L_{i+1} x\right)(t)>0$ are valid (If there exists no $i$ with this property, then the boundedness of the function $L_{0} x$ follows immediately from Lemma 1). Then from condition 4 of Theorem 2 and from Lemma 1 it follows that $n-i \equiv 0(\bmod 2)$ and $\lim _{t \rightarrow \infty}\left(L_{i} x\right)(t)>0$. From the last inequality and from (3) we derive that eventually the following inequality is valid

$$
\frac{\left(L_{0} x\right)(t)}{R_{i}(t)} \geq c>0, \quad \text { i.e. } x(t) \geq \frac{c R_{i}(t)}{\overline{\tau_{0}(t)}}
$$

We apply Lemma 1 and obtain that $\lim _{t \rightarrow \infty}\left(L_{i+1} x\right)(t)<\infty$. Then from (2) it follows that

$$
\left|\left(\psi_{i+1} L_{n} x\right)(t)\right|<\infty
$$

From the fact the eventually

$$
\left|\left(L_{n} x\right)(t)\right|=(A x)(t) \geq\left(A \frac{c R_{i}}{\tau_{0}}\right)(t)
$$


and from inequalities (11) and (12) it follows that

$$
\left|\left(\psi_{i+1} A \frac{c R_{i}}{\tau_{0}}\right)(t)\right|<\infty
$$

which contradicts condition (10).

Corollary 1. Let the conditions of Theorem 2 hold and let $n$ be even. Then all solutions of equation (1) oscillate.

Theorem 3. Let the following conditions hold:

1. Conditions $(H)$ are met.

2. Condition 2 of Theorem 1 holds.

3. $\delta=-1$.

4. For any integer $i \in[0, n-1]$ which is odd if $n$ is even and vice versa, and for any constant $c \in \mathbf{R} \backslash\{0\}$ the following relation is eventually valid

$$
\left|\left(\psi_{\min \{i+1, n-1\}} A \frac{c R_{i}}{\tau_{0}}\right)(t)\right|=\infty
$$

Then for any nonoscillating solution $x$ of equation (1) just one of the following assertions is valid:

1.

$\lim _{t \rightarrow \infty}\left(L_{j} x\right)(t)=0, j=0, \ldots, n-1$ and then $n$ is even.

2.

$$
\begin{gathered}
\left|\lim _{t \rightarrow \infty}\left(L_{j} x\right)(t)\right|=\infty, j=0, \ldots, n-1 \quad \text { and then } \\
\frac{R_{n-1}(t)}{\tau_{0}(t)}=o(x(t)) \text { as } t \rightarrow \infty
\end{gathered}
$$


Proof. Let $x$ be an eventually nonzero nonoscillating solution of equation (1) for $\delta=-1$. Without loss of generality we can assume that $x(t) \geq 0$. Then $\left(L_{n} x\right)(t) \geq 0$ eventually. Denote by $i(i=0, \ldots, n-1)$ the least integer such that the inequalities $\left(L_{i} x\right)(t) \geq 0$ and $\left(L_{i+1} x\right)(t) \geq$ 0 are eventually valid (If there exists no such integer $i \in[0, n-1]$, then from Lemma 1 it follows that the function $L_{0} x$ is bounded. Then assertion 1 of Theorem 3 follows from Theorem 1). From condition 4 of Theorem 3 it follows that $n-i \equiv 1(\bmod 2)$. If $i=n-1$, then from Lemma 1 we obtain that $\lim _{t \rightarrow \infty}\left(L_{n-1} x\right)(t)>0$.

Suppose that $\lim _{t \rightarrow \infty}\left(L_{n-1} x\right)(t)<\infty$. Then eventually

$$
\left(\psi_{n-1} A x\right)(t)=\left(\psi_{n-1} L_{n} x\right)(t)<\infty
$$

But from assertion 6 of Lemma 1 it follows that eventually

$$
\frac{\left(L_{0} x\right)(t)}{R_{n-1}(t)} \geq c>0 \quad \text {, i.e. } x(t) \geq \frac{c R_{n-1}(t)}{\tau_{0}(t)}
$$

From (14) and (15) we obtain a contradiction with condition (13). Hence $\lim _{t \rightarrow \infty}\left(L_{n-1} x\right)(t)=\infty$. Then $\lim _{t \rightarrow \infty}\left(L_{j} x\right)(t)=\infty$ for $j=0, \ldots, n-1$. The relation $\frac{R_{n-1}(t)}{\tau_{0}(t)}=o(x(t))$ as $t \rightarrow \infty$ follows from (3) for $i=n-1$. For $i \leq n-3$ we get to a contradiction by arguments analogous to those in the proof of Theorem 2 .

Remark 2. In Theorems 1.3 let condition $\mathrm{H} 6$ be replaced by the weaker condition $\mathrm{H} 7$ :

H7: If $x \in D_{n}$ and $\sup \{t: x(t)=0\}<\infty$, then the function $A x$ is eventually nonzero.

Then the assertions of Theorems 1-3 will be valid if we replace in them "nonoscillating solution" by "non-weakly-oscillating solution". 


\section{SOME PARTICULAR REALIZATIONS OF THE OPE- RATOR $A$}

Theorem 4. Let the following conditions hold:

$$
\text { 1. } \begin{aligned}
(A x)(t)= & \max _{p_{1}(t) \leq s \leq q_{1}(t)} F_{1}\left(t_{1}, g_{1}\left(d_{1}(s) x\left(h_{1}(s)\right)\right)+\right. \\
& +\min _{p_{2}(t) \leq s \leq q_{2}(t)} F_{2}\left(t, g_{2}\left(d_{2}(s) x\left(h_{2}(s)\right)\right)\right.
\end{aligned}
$$

where for each $i=1,2$

$$
\begin{gathered}
p_{i}, q_{i}, d_{i}, h_{i} \in C\left(\left[t_{0}, \infty\right) ; R\right) \\
\lim _{t \rightarrow \infty} p_{i}(t)=\lim _{t \rightarrow \infty} h_{i}(t)=\infty, d_{i}(t)>0 \\
p_{i}(t) \leq q_{i}(t)
\end{gathered}
$$

$g_{i} \in C(R, R)$ are nondecreasing functions and $\operatorname{sgn} g_{i}(u)=\operatorname{sgn}(u)$

$$
F_{i} \in C\left(\left[t_{0}, \infty\right) \times R ; R\right)
$$

$F_{i}(t, u)$ are nondecreasing functions with respect to $u$.

2. Conditions $\mathrm{H} 1$ - $\mathrm{H} 4$ hold.

3. Condition 2 of Theorem 1 holds.

Then for the existence of an eventually nonzero nonoscillating solution $x$ of the equation

$$
\begin{gathered}
\left(L_{n} x\right)(t)+\delta\left(\operatorname { m a x } _ { p _ { 1 } ( t ) \leq s \leq q _ { 1 } ( t ) } F _ { 1 } \left(t, g_{1}\left(d_{1}(s) x\left(h_{1}(s)\right)\right)+\right.\right. \\
\min _{p_{2}(t) \leq s \leq q_{2}(t)} F_{2}\left(t, g_{2}\left(d_{2}(s) x\left(h_{2}(s)\right)\right)\right)=0
\end{gathered}
$$

for which $L_{0} x$ is a bounded function it is necessary for $\delta=1(\delta=$ -1) than $n$ be an odd (even) number. For these solutions the following relations are valid

$$
\lim _{t \rightarrow \infty}\left(L_{i} x\right)(t)=0 \quad \text { and }
$$




$$
\operatorname{sgn}\left(L_{i} x\right)(t)=(-1)^{i} \operatorname{sgn} x(t), i=0, \ldots, n-1 \text {. }
$$

Theorem 5. Let the following conditions hold:

1. Condition 1 of Theorem \& holds.

2. Conditions $\mathrm{H} 1-\mathrm{H} 4$ hold.

3. Conditions 2, 3 and 4 of Theorem 2 are met.

Then the assertion of Theorem \& is valid without the requirement for boundedness of the function $L_{0} x$.

Theorem 6. Let the following conditions hold:

1. Condition 1 of Theorem \& holds.

2. Conditions $\mathrm{H}_{1-\mathrm{H}} 4$ hold.

3. Conditions 3 and 4 of Theorem 3 are met.

Then for any nonoscillating solution of equation (17) just one of the following assertions is valid:

1. $\lim _{t \rightarrow \infty}\left(L_{j} x\right)(t)=0, j=0, \ldots, n-1$ and then $n$ is even.

2. $\left|\lim _{t \rightarrow \infty}\left(L_{j} x\right)(t)\right|=\infty, j=0, \ldots, n-1$ and then

$$
\frac{\underline{R_{n-1}(t)}}{\tau_{0}(t)}=o(x(t)) \quad \text { as } t \rightarrow \infty
$$

Theorems 4,5 and 6 are particular cases of Theorems 1, 2 and 3, respectively.

Remark 3. If only one of the two addends enter the right-hand side of (16), then the operator $A$ satisfies conditions H3-H5 and H7.

Theorem 7. Let the following conditions hold:

$$
\text { 1. }(A x)(t)=F\left(t, \int_{p(t)}^{q(t)} K(t, s, x(t), x(s)) d_{s} \tau(t, s)\right)
$$


where:

a) The function $F$ satisfies condition 1 of Theorem 4.

b) $p, q \in C\left(\left[t_{0}, \infty\right) ; R\right), \lim _{t \rightarrow \infty} p(t)=\infty, p(t)<q(t)$

c) $K \in C\left(\left[t_{0}, \infty\right)^{2} \times R^{2}, R\right)$. The function $K(t, s, u, v)$ is nondecreasing with repect to $u$ and to $v$.

d) $\operatorname{sgn} K(t, s, u, o)=\operatorname{sgn} u$ $\operatorname{sgn} K(t, s, o, v)=\operatorname{sgn} v$

e) For any $t \in\left[t_{0}, \infty\right)$ the function $s \rightarrow \tau(t, s)$ is increasing.

f) The functions $t \rightarrow \tau(t, p(t))$ and $t \rightarrow \tau(t, q(t))$ are continuous and for $t \in\left[t_{0}, \infty\right)$ the following relation is valid

$$
\lim _{t^{\prime} \rightarrow t} \int_{\max \left\{p(t), p\left(t^{\prime}\right)\right\}}^{\min \left\{q(t), q\left(t^{\prime}\right)\right\}}\left|\tau\left(t^{\prime}, s\right)-\tau(t, s)\right| d s=0
$$

2. Conditions H1-H4 are met.

3. Condition 2 of Theorem 1 holds.

Then for the existence of an eventually nonzero nonoscillating solution $x$ of the equation

$$
\left(L_{n} x\right)(t)+\delta \cdot F\left(t, \int_{p(t)}^{q(t)} K(t, s, x(t), x(s)) d_{s} \tau(t, s)\right)=0
$$

for which $L_{0} x$ is a bounded function it is necessary for $\delta=1(\delta=$ -1) that $n$ be an odd (even) number. For these solutions the following relations are valid

$\lim _{t \rightarrow \infty}\left(L_{i} x\right)(t)=0$ and $\operatorname{sgn}\left(L_{i} x\right)(t)=(-1)^{i} \operatorname{sgn} x(t), i=0, \ldots, n-1$ 
Theorem 8. Let the following conditions hold:

1. Condition 1 of Theorem 7 holds.

2. Conditions H1-H4 hold.

3. Conditions 2, 3 and 4 of Theorem 2 are met.

Then the assertion of Theorem 7 is valid without the requirement for boundedness of the function $L_{0} x$.

Theorem 9. Let the following conditions hold:

1. Condition 1 of Theorem 7 holds.

2. Conditions $\mathrm{H} 1-\mathrm{H} 4 \mathrm{~h}$ hold.

3. Conditions 3 and 4 of Theorem 3 are met.

Then for any nonoscillating solution $x$ of equation (19) just one of the following assertions is valid:

1. $\lim _{t \rightarrow \infty}\left(L_{j} x\right)(t)=0, j=0, \ldots, n-1$ and then $n$ is even.

2. $\left|\lim _{t \rightarrow \infty}\left(L_{j} x\right)(t)\right|=\infty, j=0, \ldots, n-1$ and then

$$
\frac{R_{n-1}(t)}{\tau_{0}(t)}=o(x(t)) \quad \text { as } t \rightarrow \infty
$$

Theorems 7,8 and 9 are particular cases of Theorems 1,2 and 3, respectively.

Remark 4. If the operator $A$ defined by (18) satisfies condition 1 of Theorem 7 but conditions $1 \mathrm{~d}$ and $1 \mathrm{e}$ are replaced by the conditions

$$
\begin{array}{ll}
K(t, s, u, v)>0 & \text { for } u>0, v>0 \\
K(t, s, u, v)<0 & \text { for } u<0, v<0
\end{array}
$$

and 
the function $s \rightarrow \tau(t, s)$ is nondecreasing and nonconstant in the interval $[p(t), q(t)]$ respectively, then the operator $A$ satisfies conditions $\mathrm{H} 3-\mathrm{H} 5$ and $\mathrm{H} 7$.

\section{References}

[1] Kigurazde, I.T.: Some singular boundary value problems for ondinary differential equations. Izd. Tbilisi State Univ., Tbilisi, 1975 (in Russian).

[2] Ladde, G.S., Lakshmikantham, V. and Zhang, B.G.: Oscillation theory of differential equations with deviating arguments. Pure and Applied Mathematics, Vol. 110, Marcel Dekker, 1987.

[3] Mishev, D.P. and Bainov, D.D.: Some properties of the nonoscillating solutions of functional differential equations of $n$-th order. Rendiconti del Circolo Matematico di Palermo, 35 (1986), 233-243.

[4] Myshkis, A.D.: Linear Differential Equations with Retarded Argument. M.-L., Gostekhizdat; 1951 (in Russian).

[5] Philos, Ch.G.: Oscillatory and asymptotic behavior of the bounded solutions of differential equations with deviating arguments. Hiroshima Math. J., Vol. 8, No. 1 (1978), 31-48.

[6] Philos, Ch.G.: Oscillatory and asymptotic behavior of all solutions of differential equations with deviating arguments. Proceedings of the Royal Society of Edinburgh, 81A (1978), 195-210.

[7] Philos, Ch.G.: On the asymptotic behavior of the nonoscillatory solutions of differential equations with deviating arguments. Math. Nachr., 113 (1983), 107-128.

[8] Philos, Ch.G. and Staikos, V.A.: A basic asymptotic criterion for differential equations with deviating arguments and its applications to the nonoscillation of linear ordinary equations, Nonlinear Analysis, Theory, Methods and Applications, Vol. 6, No. 10 (1982), 1095-1113. 
[9] Philos, Ch.G., Sficas, Y.G. and Staikos, V.A.: Some results on the asymptotic behavior of nonoscillatory solutions of differential equations with deviating arguments. J. Austral. Math. Soc. (Series A) 32 (1982), 295-317.

South-West University

"Neophyte Rilski"

Blagoevgrad

BULGARIA

Railway Transport

Engineering Institute

Moscow

RUSSIA
Filial Technical University

Sliven

BULGARIA

Recibido: 15 de abril de 1993 